\title{
Design of photonic microcavities in hexagonal boron nitride
}

\author{
Sejeong Kim ${ }^{*}$, Milos Toth and Igor Aharonovich
}

\author{
Letter \\ Address: \\ School of Mathematical and Physical Sciences, University of \\ Technology Sydney, Ultimo, NSW 2007, Australia \\ Email: \\ Sejeong Kim* - Sejeong.Kim-1@uts.edu.au \\ * Corresponding author \\ Keywords: \\ anisotropic optical materials; boron nitride; photonic crystals
}

\author{
Beilstein J. Nanotechnol. 2018, 9, 102-108. \\ doi:10.3762/bjnano.9.12
}

Received: 18 August 2017

Accepted: 12 December 2017

Published: 09 January 2018

This article is part of the Thematic Series "Light-Matter interactions on the nanoscale".

Guest Editor: M. Rahmani

(c) 2018 Kim et al.; licensee Beilstein-Institut.

License and terms: see end of document.

\begin{abstract}
We propose and design photonic crystal cavities (PCCs) in hexagonal boron nitride (hBN) for diverse photonic and quantum applications. Two dimensional (2D) hBN flakes contain quantum emitters which are ultra-bright and photostable at room temperature. To achieve optimal coupling of these emitters to optical resonators, fabrication of cavities from hBN is therefore required to maximize the overlap between cavity optical modes and the emitters. Here, we design 2D and 1D PCCs using anisotropic indices of $\mathrm{hBN}$. The influence of underlying substrates and material absorption are investigated, and spontaneous emission rate enhancements are calculated. Our results are promising for future quantum photonic experiments with hBN.
\end{abstract}

\section{Introduction}

Hexagonal boron nitride (hBN) has recently emerged as an interesting platform for nanophotonics. This is mainly due to its promising hyperbolic properties $[1,2]$ as well as the ability to host a range of single photon emitters (SPEs) that are of great interest for a myriad of nanophotonics and quantum photonic applications [3-10]. However, to further study light matter interactions based on the hBN SPEs, and to realize integrated nanophotonics systems, coupling of the emitters to optical cavities is essential [11-15].

Typically, SPEs can be coupled to optical cavities using two general approaches. The cavity is either made from the material that hosts the emitter (monolithic approach), or the emitter is coupled to a cavity made from a foreign material - also known as a hybrid approach [16]. The former process is preferred when attempting to maximize the field overlap between the emitters and the cavity modes, and is often employed when using materials that are amenable to scalable nanofabrication protocols, such as gallium arsenide or silicon [17,18], and more recently diamond and silicon carbide $[19,20]$. The hybrid approach is easier from the fabrication point of view but is inherently limited by the fact that the electric field maxima of optical modes are situated within the cavities, and optimal coupling therefore remains a challenge. 
The optical properties of hBN make it an attractive candidate for a monolithic cavity system. In particular, hBN has a wide bandgap of $\approx 6 \mathrm{eV}$ which makes it transparent in the visible spectral range that contains the zero phonon lines (ZPLs) of a range of ultra-bright emitters [21]. Furthermore, hBN has properties which are desirable for micro-resonators such as a high chemical stability and an excellent thermal conductivity $[22,23]$.

In this work, we propose to use $\mathrm{hBN}$ for the fabrication of photonic crystal cavities (PCCs). We design two dimensional (2D) PCCs and show that they have high quality-factor ( $Q$-factor) resonances in the visible spectral range, which overlap with the ZPLs of SPEs in hBN [24]. We further optimize the structures and model $1 \mathrm{D}$ nanobeam photonic crystals that exhibit a $Q$-factor in excess of $\approx 20,000$. In the light of recent progress in direct-write etching of hBN [25], our results are promising for realization of high $Q$ cavities and monolithic coupled systems made from this material.

\section{Results and Discussion}

We begin with a 2D photonic crystal that contains a line defect cavity. The L3 cavity has been widely investigated because it was the first to exceed an experimental $Q$-factor of 10,000 [26,27]. In this study, we used a commercial finite-difference time-domain (FDTD) software package (Lumerical Inc.). The 3D FDTD simulation domain for 2D (1D) photonic crystal was $7 \mu \mathrm{m} \times 7 \mu \mathrm{m} \times 1.2 \mu \mathrm{m}(11 \mu \mathrm{m} \times 2 \mu \mathrm{m} \times 2 \mu \mathrm{m})$ which is discretised using uniform spatial and temporal grids of $15 \mathrm{~nm}$ and $0.03 \mathrm{fs}$. Birefringence of hBN is accounted for in our study by including both ordinary $\left(n_{\mathrm{x}}=n_{\mathrm{y}}=1.72\right)$ and extraordinary $(n=1.84)$ indices in the 3D FDTD method models. Figure 1a shows the shape and the size of typical hBN flakes prepared by scotch-tape exfoliation [9]. The lateral flake size varies from a few micrometers to few tens of micrometers while thickness varies from few tens of nanometers to a few micrometers, which is sufficient for the fabrication of practical photonic crystal cavities. The parameters used to define an L3 cavity are shown in Figure $1 b$. The cavity consists of a free-standing slab with a triangular photonic lattice with periodicity ' $a$ '. The air hole radius in the mirror region and the radius of two side air holes are fixed at $0.33 a$ and $0.22 a$, respectively. By tuning the periodicity ' $a$ ', one can tune the resonant wavelength whilst preserving the $Q$-factor. In this study, we used $a=270 \mathrm{~nm}$ and $t=280 \mathrm{~nm}$ to place the fundamental mode within the typical emission range of SPEs in hBN (550-700 nm). Two air holes at
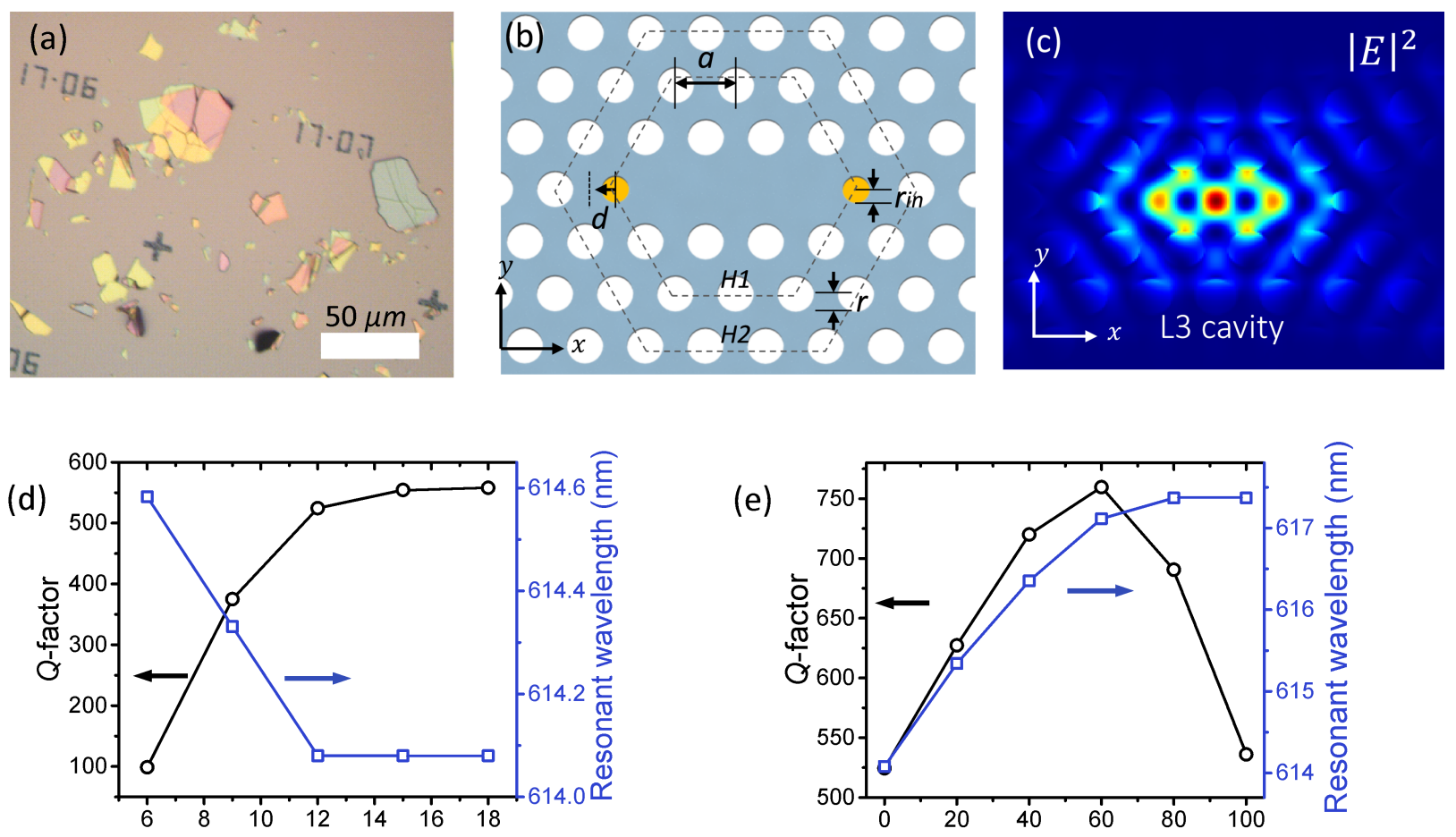

Number of photonic crystal layers, $H$

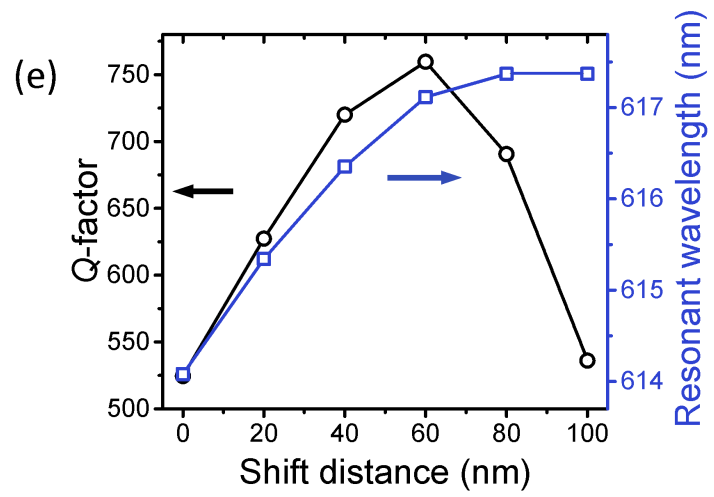

Figure 1: (a) Optical image of exfoliated hBN flakes. (b) Schematic of a 2D photonic crystal with an L3 cavity. The geometric parameters are the following: the period ' $a$ ', the radius of air holes ' $r$ ', the radius of two side air holes ' $r$ in', the shift distance of the side air holes ' $d$ ', the thickness of the hBN slab ' $t$ ' and the number of photonic crystal layers ' $H$ '. (c) Three-dimensional FDTD simulation of the electric field intensity profile of the fundamental mode of the L3 cavity. $Q$-factor and the resonant wavelength calculated as a function of (d) the number of photonic crystal layers ' $H$ ', and (e) the shift distance of the two side air holes ' $d$ '. 
the side of the cavity (yellow circles in Figure 1b) are decreased in size to reduce radiation losses [28]. For the purpose of design simplicity, we adopt tuning of the side air holes as the only means used to increase the $Q$-factor. Lastly, the number of photonic crystal layers comprising the cavity is denoted by ' $H$ ' which act as a photonic mirror and the thickness of the hBN slab by ' $t$ '. The electric field intensity pattern of the L3 cavity calculated using 3D FDTD simulation is shown in Figure 1c. This is the lowest energy mode in the L3 cavity which is the most widely studied of high- $Q 2 \mathrm{D}$ photonic cavities.

To optimize the design, we start by increasing the number of photonic crystal layers $H$, in order to increase the photonic mirror strength and to reduce the in-plane loss of the cavity. As shown in Figure 1d, the $Q$-factor of the mode starts to saturate at $H \approx 12$ because the $Q$-factor is limited not only by the in-plane component, but also by radiation loss. Considering both the $Q$-factor and scaling of the simulation time with domain size, we fixed $H$ at 12 for subsequent modelling.
Radiation losses can be reduced by optimizing the side air hole positions, as is shown in Figure 1e, which reveals that the cavity $Q$-factor is greatest at a shift distance of $60 \mathrm{~nm}$. Note that the mode resonance red shifts with increasing side hole separation due to an effective increase in cavity length.

Photonic crystal cavities with line defects can be described as Fabry-Pérot resonators [29]. Hence, the $Q$-factor can be enhanced by increasing the cavity length as for a Fabry-Pérot resonator [30], which can be tuned by varying number of missing air holes. Figure 2a shows the $Q$-factors of L3, L7, and L11 cavities with a slab thickness of $280 \mathrm{~nm}$. The electric field intensity profiles of L7 and L11 are also shown in the figure (and that of the L3 cavity is shown in Figure 1c). The $Q$-factor of the fundamental mode increases with effective cavity length. For the L11 cavity, we additionally calculated the effect of the thickness of the $2 \mathrm{D}$ photonic crystal slab as is shown in Figure $2 \mathrm{~b}$. The simulated slab thickness is varied up to $300 \mathrm{~nm}$ since that is a realistic thickness of typical hBN flakes prepared

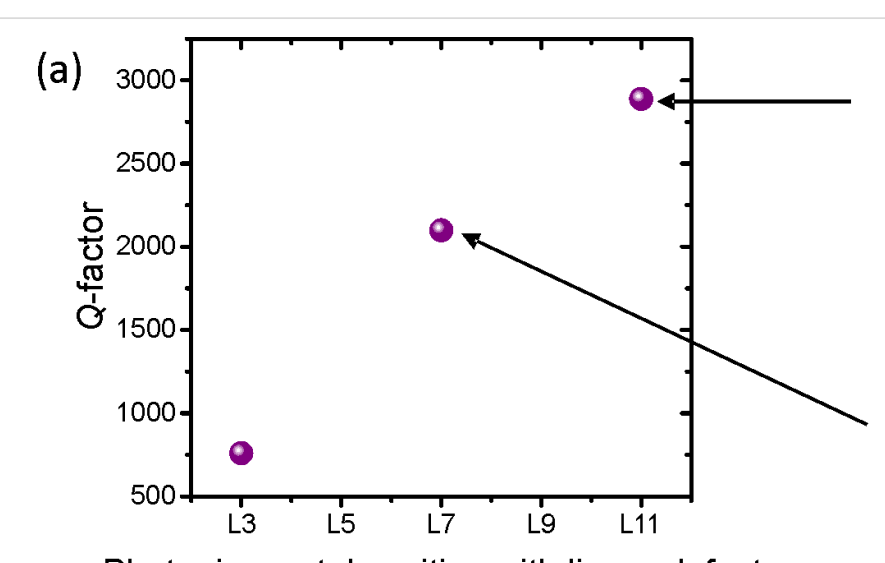

Photonic crystal cavities with linear defects
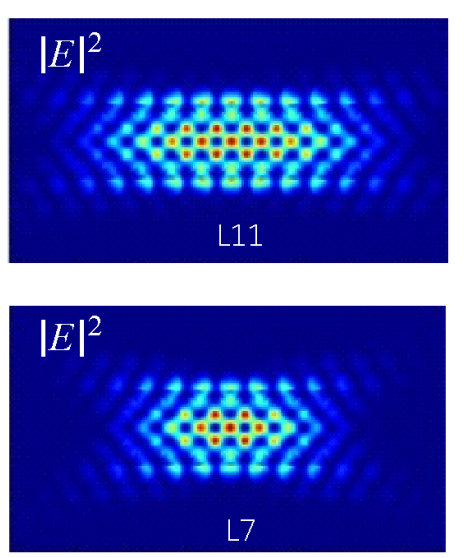

(b)

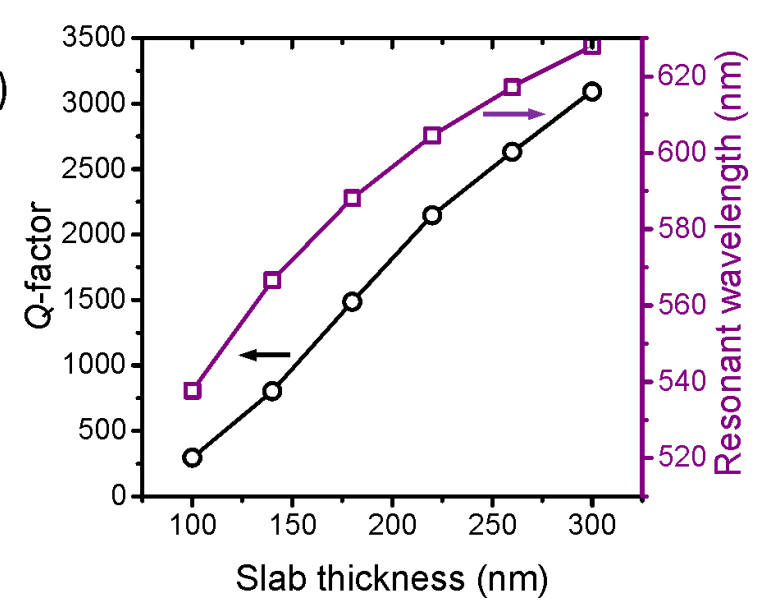

(c)

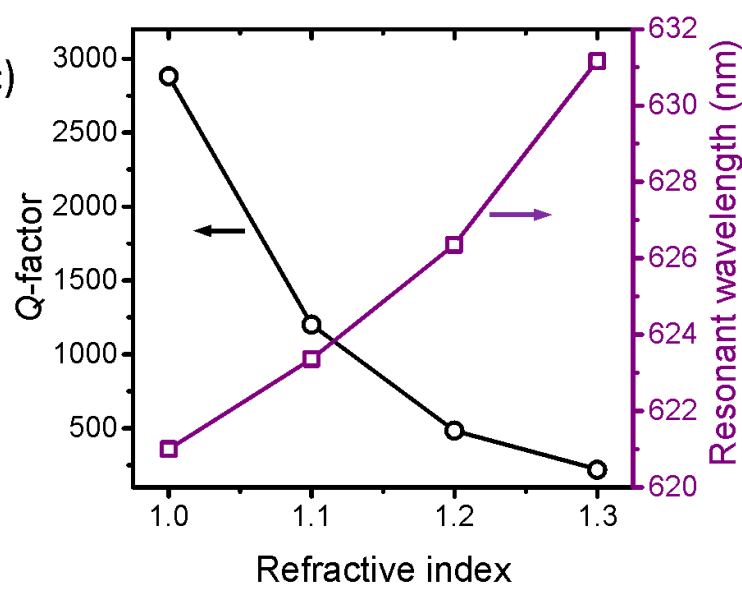

Figure 2: (a) Q-factors of various 2D photonic crystal cavities with an increasing number of linear defects, and the electric field intensity profiles of $L 7$ and L11 cavities. Q-factor and resonant wavelength plotted as a function of (b) the slab thickness and (c) the refractive index of an underlying substrate. 
by the scotch tape exfoliation method. Thicker slabs exhibit stronger light confinement, which results in higher $Q$-factors.

We also modelled the effect of the refractive index of an underlying substrate, as is shown in Figure 2c. Because the refractive index of $\mathrm{hBN}$ is relatively low compared to that of typical semiconductors, the increase in substrate index greatly degrades the $Q$-factor of the L11 cavity (and a similar effect is expected for the other cavities as well). This is, however, not a significant problem as the transfer of hBN flakes onto holey substrates is a straightforward process. Furthermore, use of aerogel material that is currently commercially available is another option to achieve low index substrates. We note that the overall $Q$-factor is lower than in typical semiconductor $2 \mathrm{D}$ photonic crystals due to the relatively low refractive index of $\mathrm{hBN}$.

Next we investigate one dimensional nanobeam photonic crystal structures. 1D PCCs are advantageous in that they can easily have full photonic bandgap between the first and the second lowest photonic energy bands even when the effective index contrast is low [31]. The combination of a high $Q$-factor and a low refractive index enables a broad range of applications such as flexible photonic crystal devices and high figure of merit sensors [32]. Figure 3a shows the electric field intensity profile of the fundamental mode. The cavity is designed by modulating the periodicity whilst fixing the air hole radius [33]. The structure consists of a total of 31 air holes, 15 of which are modulated to create a cavity in the center, and the remaining 8 on each end act as photonic mirrors. In Figure $3 b$, we set the periodicity in the mirror region to $260 \mathrm{~nm}$ while the air hole radius and the nanobeam width are fixed at $70 \mathrm{~nm}$ and $300 \mathrm{~nm}$, respectively. By increasing the thickness of the slab from $200 \mathrm{~nm}$ to $300 \mathrm{~nm}$, the $Q$-factor increases, as is seen in Figure $3 \mathrm{~b}$, and $Q$-factors in excess of 20,000 can be realized. These values are achieved even without optimization of the remaining structural parameters, and are over an order of magnitude greater than the maximum $Q$-factor of a low-index $2 \mathrm{D}$ cavity. Next, we fix the thickness at $280 \mathrm{~nm}$ and tune the structural parameters to further increase the $Q$-factor of the mode. Figure $3 \mathrm{c}$ shows the $Q$-factor plotted as a function of the nanobeam width ' $w$ ', showing that the $Q$-factor has a maximum at a width of $320 \mathrm{~nm}$ [34]. Introducing a substrate to the (a)
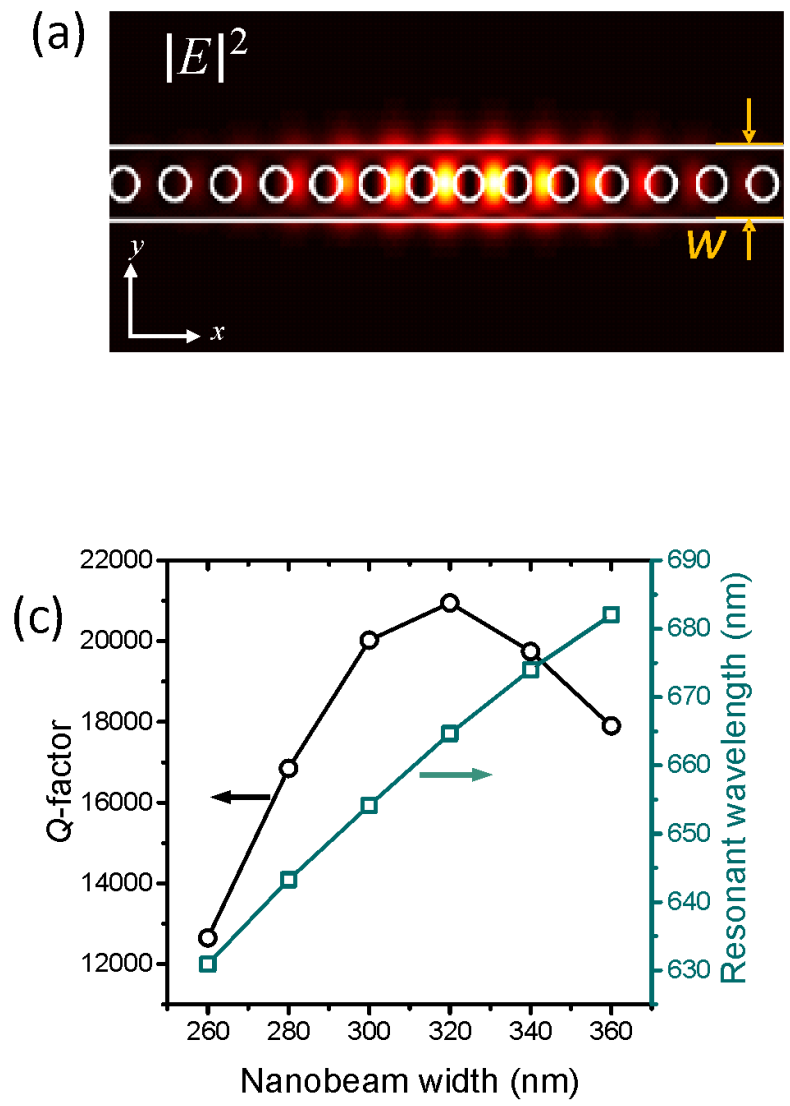

(b)

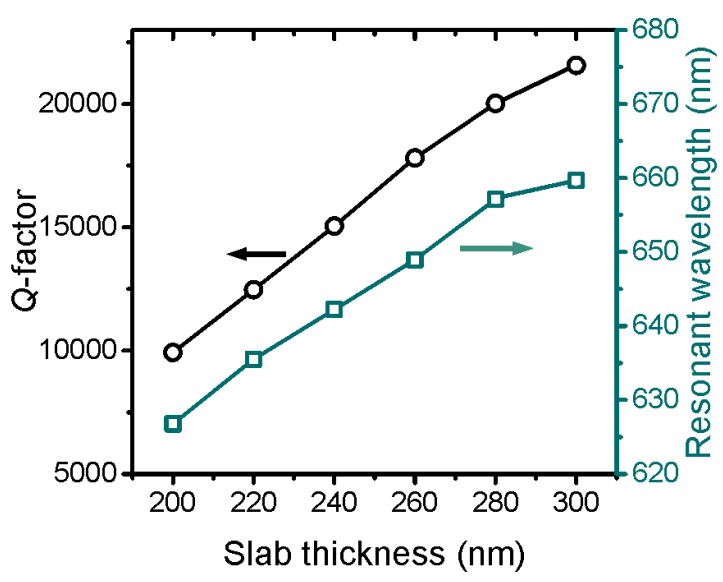

(d)

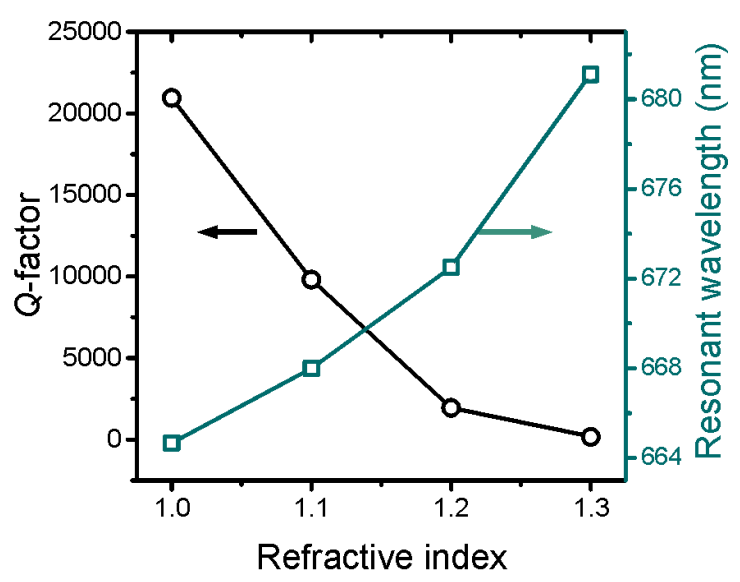

Figure 3: (a) Calculated electric field intensity distribution for a 1D photonic crystal cavity. 3D FDTD simulation of $Q$-factor versus (b) slab thickness, (c) nanobeam width ' $w$ ', and (d) refractive index of the substrate, respectively. 
nanobeam degrades the $Q$-factor as is shown in Figure $3 \mathrm{~d}$. A free-standing structure is preferred, as in the case of the 2D photonic crystals presented earlier.

We also consider the absorption losses in the cavity. Figure $4 \mathrm{a}$ shows the effect of the absorption by the cavity material. Many FDTD modelling studies of semiconductor photonic crystals include only the real part of the complex refractive index as a structural input which assumes that the material is transparent in the simulated wavelength regime. However, in the case of practical situations in which the cavity material exhibits finite absorption, the imaginary part should also be accounted for. The $Q$-factor of the fundamental mode in a free-standing nanobeam with a beam-width $(w)$ of $320 \mathrm{~nm}$ and a slab thickness $(t)$ of $280 \mathrm{~nm}$ is calculated as a function of the imaginary refractive index. An increase in imaginary refractive index (i.e., an increase in material absorption) causes the $Q$-factor to decrease significantly. Note that realistic $Q$-factor is determined by $1 / Q=1 / Q_{\text {ideal }}+1 / Q_{\text {abs }}\left(Q_{\text {ideal }}: Q\right.$-factor with lossless material, $Q_{\text {abs }}: Q$-factor with absorption losses) [35], which indicates the material loss restricts the maximum $Q$-factor that can obtained through experiment. Therefore, including imaginary refractive index for $Q$-factor calculation provides practical $Q$-factors.

Finally, we discuss the expected Purcell enhancement due to coupling of emitters to cavity modes. Figure $4 \mathrm{~b}$ shows the spontaneous emission rate $\left(\gamma_{\mathrm{sp}}\right)$ of a quantum emitter in the nanobeam cavity relative to an emitter in free space $\left(\gamma_{\mathrm{sp}}^{0}\right)$. The Purcell enhancement $\left(\gamma_{\mathrm{sp}} / \gamma_{\mathrm{sp}}^{0}\right)$ was found at any given spatial position by calculating the radiated power enhancement of the quantum emitter relative to that in free space. Because the fundamental mode of the nanobeam has a maximum in the high index region, the $Q$-factor is calculated across the dashed line $(y$-axis) shown in the inset (i.e., along the width of the 1D PCC). The dipole emitter in the simulation is $y$-polarized to match the polarization of the optical mode. The Purcell enhancement has a maximum in the center of the nanobeam where the electric field intensity is the greatest. The expected Purcell enhancement is greater than 100 over a range of more than $200 \mathrm{~nm}$ along the $y$-axis, as is seen in Figure $4 \mathrm{~b}$, which relaxes the experimental conditions to precisely position the SPE in the cavity. For a realistic case, with a SPE that exhibits a ZPL at $670 \mathrm{~nm}$ that is on resonance with the cavity mode, even moderate $Q$ values of 20,000 ( $\approx 20 \mathrm{GHz}$ linewidth), will yield a Purcell enhancement of $\approx 530$. To realize the high Purcell enhancement experimentally, techniques for precise positioning of SPEs to the maximum intensity of the cavity mode are required. Two different approaches can suggest solutions to this problem. The first is to find pre-existing single emitters and post-fabricate optical cavities around them. The other approach is to determin-
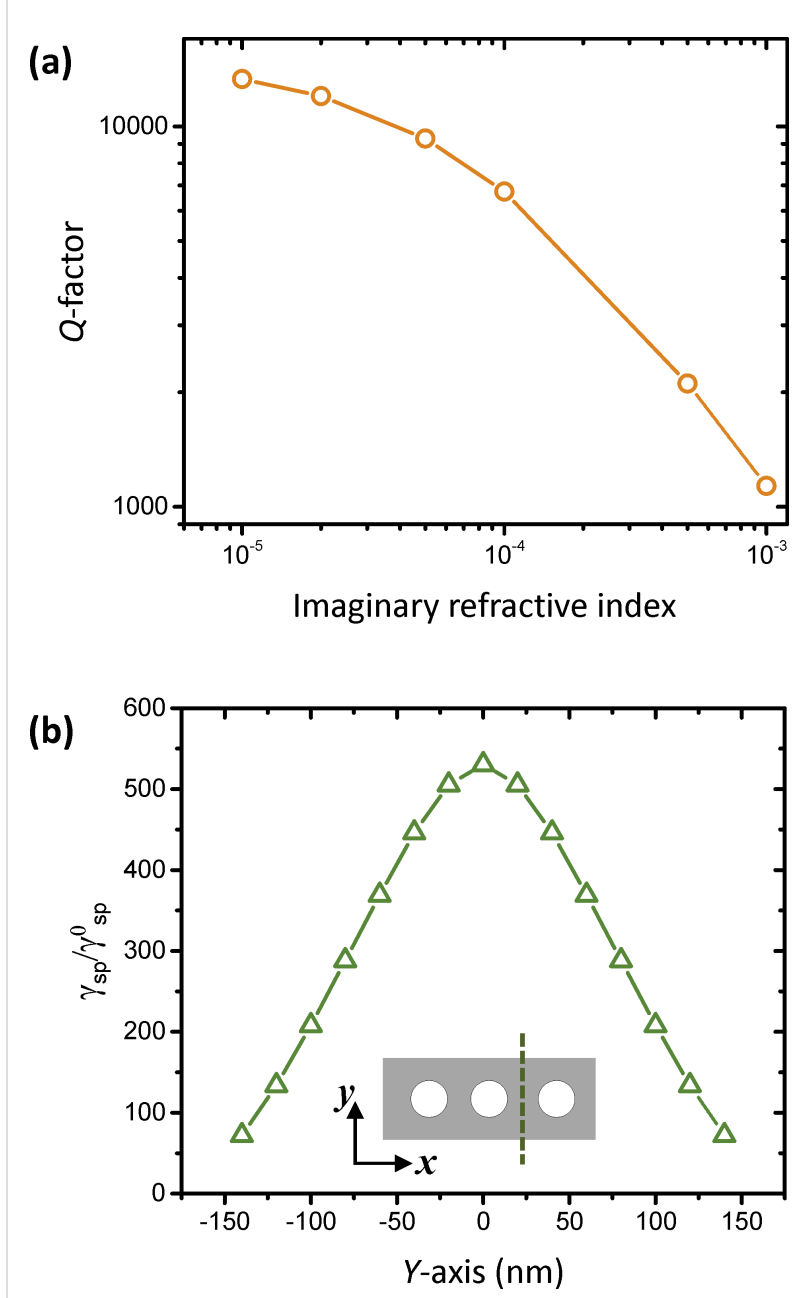

Figure 4: (a) Q-factor of the fundamental mode in a 1D photonic crystal cavity versus the imaginary refractive index coefficient of the material. (b) Spontaneous emission rate enhancement of a dipole emitter plotted as a function of distance across the 1D photonic crystal nanobeam cavity.

istically create emitters in desired locations. Recent studies of hBN single emitters show promising results for both approaches [36,37].

\section{Conclusion}

In summary, we described and optimized a number of $2 \mathrm{D}$ and 1D PCC designs in free-standing and supported hBN layers. Linear defect cavities were studied as representatives of 2D photonic crystals. Period modulation of a 1D nanobeam was used to achieve a theoretical $Q$-factor in excess of 20,000 simply by modulating the beam width of the structure. The effect of the imaginary refractive index on the $Q$-factor of a nanobeam was simulated, as was the Purcell factor, showing a strong interaction between a dipole emitter and the optical mode. The designs and analyses of the hBN photonic cavities presented in this work will pave a way to a broad range of ap- 
plications enabled by integrated photonic circuits based on $2 \mathrm{D}$ materials.

\section{Funding}

Financial support from the Australian Research Council (DE130100592, DP140102721), the Asian Office of Aerospace Research and Development grant FA2386-17-1-4064 are gratefully acknowledged.

\section{Acknowledgements}

We thank Prof. Joshua D. Caldwell for useful discussions on $\mathrm{hBN}$ refractive index.

\section{ORCID ${ }^{\circledR}$ iDs}

Sejeong Kim - https://orcid.org/0000-0001-9836-3608

\section{References}

1. Caldwell, J. D.; Kretinin, A. V.; Chen, Y.; Giannini, V.; Fogler, M. M.; Francescato, Y.; Ellis, C. T.; Tischler, J. G.; Woods, C. R.; Giles, A. J.; Hong, M.; Watanabe, K.; Taniguchi, T.; Maier, S. A.; Novoselov, K. S. Nat. Commun. 2014, 5, No. 5221. doi:10.1038/ncomms6221

2. Dai, S.; Ma, Q.; Andersen, T.; Mcleod, A. S.; Fei, Z.; Liu, M. K.; Wagner, M.; Watanabe, K.; Taniguchi, T.; Thiemens, M.; Keilmann, F.; Jarillo-Herrero, P.; Fogler, M. M.; Basov, D. N. Nat. Commun. 2015, 6, No. 6963. doi:10.1038/ncomms7963

3. Bourrellier, R.; Meuret, S.; Tararan, A.; Stéphan, O.; Kociak, M.; Tizei, L. H.; Zobelli, A. Nano Lett. 2016, 16, 4317. doi:10.1021/acs.nanolett.6b01368

4. Chejanovsky, N.; Rezai, M.; Paolucci, F.; Kim, Y.; Rendler, T.; Rouabeh, W.; Fávaro de Oliveira, F.; Herlinger, P.; Denisenko, A.; Yang, S.; Gerhardt, I.; Finkler, A.; Smet, J. H.; Wrachtrup, J. Nano Lett. 2016, 16, 7037. doi:10.1021/acs.nanolett.6b03268

5. Feng, J.; Deschout, H.; Caneva, S.; Hofmann, S.; Lončarić, I.; Lazić, P.; Radenovic, A. arXiv 2017, No. 1706.06313. https://arxiv.org/abs/1706.06313

6. Jungwirth, N. R.; Calderon, B.; Ji, Y.; Spencer, M. G.; Flatt, M. E.; Fuchs, G. D. Nano Lett. 2016, 16, 6052. doi:10.1021/acs.nanolett.6b01987

7. Kianinia, M.; Regan, B.; Tawfik, S. A.; Tran, T. T.; Ford, M. J.; Aharonovich, I.; Toth, M. ACS Photonics 2017, 4, 768. doi:10.1021/acsphotonics.7b00086

8. Martínez, L. J.; Pelini, T.; Waselowski, V.; Maze, J. R.; Gil, B.; Cassabois, G.; Jacques, V. Phys. Rev. B: Condens. Matter Mater. Phys. 2016, 94, 121405. doi:10.1103/PhysRevB.94.121405

9. Tran, T. T.; Bray, K.; Ford, M. J.; Toth, M.; Aharonovich, I. Nat. Nanotechnol. 2016, 11, 37. doi:10.1038/nnano.2015.242

10. Tran, T. T.; Wang, D.; Xu, Z.-Q.; Yang, A.; Toth, M.; Odom, T. W.; Aharonovich, I. Nano Lett. 2017, 17, 2634. doi:10.1021/acs.nanolett.7b00444

11. Englund, D.; Fushman, I.; Faraon, A.; Vučković, J. Photonics Nanostruct. - Fundam. Appl. 2009, 7, 56. doi:10.1016/j.photonics.2008.11.008

12. Faraon, A.; Santori, C.; Huang, Z.; Acosta, V. M.; Beausoleil, R. G. Phys. Rev. Lett. 2012, 109, 033604. doi:10.1103/PhysRevLett.109.033604
13. Kuhn, A.; Ljunggren, D. Contemp. Phys. 2010, 51, 289. doi:10.1080/00107511003602990

14. Müller, K.; Fischer, K. A.; Rundquist, A.; Dory, C.; Lagoudakis, K. G.; Sarmiento, T.; Kelaita, Y. A.; Borish, V.; Vučković, J. Phys. Rev. X 2015, 5, 031006. doi:10.1103/PhysRevX.5.031006

15. Sato, Y.; Tanaka, Y.; Upham, J.; Takahashi, Y.; Asano, T.; Noda, S. Nat. Photonics 2012, 6, 56. doi:10.1038/nphoton.2011.286

16. Benson, O. Nature 2011, 480, 193. doi:10.1038/nature10610

17. Harris, N. C.; Bunandar, D.; Pant, M.; Steinbrecher, G. R.; Mower, J.; Prabhu, M.; Baehr-Jones, T.; Hochberg, M.; Englund, D. Nanophotonics 2016, 5, 456. doi:10.1515/nanoph-2015-0146

18. Santori, C.; Fattal, D.; Vučković, J.; Solomon, G. S.; Yamamoto, Y. Nature 2002, 419, 594. doi:10.1038/nature01086

19. Bracher, D. O.; Zhang, X.; Hu, E. L. Proc. Natl. Acad. Sci. U. S. A. 2017, 114, 4060. doi:10.1073/pnas.1704219114

20. Faraon, A.; Barclay, P. E.; Santori, C.; Fu, K.-M. C.; Beausoleil, R. G. Nat. Photonics 2011, 5, 301. doi:10.1038/nphoton.2011.52

21. Cassabois, G.; Valvin, P.; Gil, B. Nat. Photonics 2016, 10, 262. doi:10.1038/nphoton.2015.277

22. Lin, Y.; Connell, J. W. Nanoscale 2012, 4, 6908. doi:10.1039/c2nr32201c

23. Liu, Z.; Gong, Y.; Zhou, W.; Ma, L.; Yu, J.; Idrobo, J. C.; Jung, J.; MacDonald, A. H.; Vajtai, R.; Lou, J.; Ajayan, P. M. Nat. Commun. 2013, 4, 2541. doi:10.1038/ncomms3541

24. Tran, T. T.; Elbadawi, C.; Totonjian, D.; Lobo, C. J.; Grosso, G.; Moon, H.; Englund, D. R.; Ford, M. J.; Aharonovich, I.; Toth, M. ACS Nano 2016, 10, 7331. doi:10.1021/acsnano.6b03602

25. Elbadawi, C.; Tran, T. T.; Kolibal, M.; Šikola, T.; Scott, J.; Cai, Q.; Li, L. H.; Taniguchi, T.; Watanabe, K.; Toth, M.; Aharonovich, I.; Lobo, C. Nanoscale 2016, 8, 16182. doi:10.1039/C6NR04959A

26. El Kurdi, M.; Checoury, X.; David, S.; Ngo, T.; Zerounian, N.; Boucaud, P.; Kermarrec, O.; Campidelli, Y.; Bensahel, D. Opt. Express 2008, 16, 8780. doi:10.1364/OE.16.008780

27. Schwagmann, A.; Kalliakos, S.; Ellis, D. J. P.; Farrer, I.; Griffiths, J. P.; Jones, G. A. C.; Ritchie, D. A.; Shields, A. J. Opt. Express 2012, 20, 28614. doi:10.1364/OE.20.028614

28. Akahane, Y.; Asano, T.; Song, B.-S.; Noda, S. Nature 2003, 425, 944. doi:10.1038/nature02063

29. Kim, S.-H.; Kim, G.-H.; Kim, S.-K.; Park, H.-G.; Lee, Y.-H.; Kim, S.-B. J. Appl. Phys. 2004, 95, 411. doi:10.1063/1.1633645

30. Hunger, D.; Steinmetz, T.; Colombe, Y.; Deutsch, C.; Hänsch, T. W.; Reichel, J. New J. Phys. 2010, 12, 065038. doi:10.1088/1367-2630/12/6/065038

31. Quan, Q.; Burgess, I. B.; Tang, S. K. Y.; Floyd, D. L.; Loncar, M. Opt. Express 2011, 19, 22191. doi:10.1364/OE.19.022191

32. Kim, S.; Kim, H.-M.; Lee, Y.-H. Opt. Lett. 2015, 40, 5351. doi:10.1364/OL.40.005351

33. Kim, S.; Ko, H.; Lee, C.; Kim, M.; Kim, K. S.; Lee, Y.-H.; Shin, K.; Cho, Y.-H. Adv. Mater. 2016, 28, 9765. doi:10.1002/adma.201603368

34. Kim, S.; Ahn, B.-H.; Kim, J.-Y.; Jeong, K.-Y.; Kim, K. S.; Lee, Y.-H. Opt. Express 2011, 19, 24055. doi:10.1364/OE.19.024055

35. Kreuzer, C.; Riedrich-Möller, J.; Neu, E.; Becher, C. Opt. Express 2008, 16, 1632. doi:10.1364/OE.16.001632

36. Kianinia, M.; Bradac, C.; Wang, F.; Sontheimer, B.; Tran, T. T.; Nguyen, M.; Kim, S.; Xu, Z.-Q.; Jin, D.; Schell, A. W.; Lobo, C. J.; Aharonovich, I.; Toth, M. arXiv 2017, No. 1709.08683. https://arxiv.org/abs/1709.08683 
37.Xu, Z.-Q.; Elbadawi, C.; Tran, T. T.; Kianinia, M.; Li, X.; Liu, D.;

Hoffman, T. B.; Nguyen,, M.; Kim, S.; Edgar, J. H.; Wu, X.; Song, L.;

Ali, S.; Ford, M. J.; Toth, M.; Aharonovich, I. arXiv 2017,

No. 1710.07010 .

https://arxiv.org/abs/1710.07010

\section{License and Terms}

This is an Open Access article under the terms of the Creative Commons Attribution License

(http://creativecommons.org/licenses/by/4.0), which permits unrestricted use, distribution, and reproduction in any medium, provided the original work is properly cited.

The license is subject to the Beilstein Journal of

Nanotechnology terms and conditions:

(http://www.beilstein-journals.org/bjnano)

The definitive version of this article is the electronic one which can be found at:

doi:10.3762/bjnano.9.12 